\title{
Analysis of clinical \& radiological findings in children with acute wheeze
}

\author{
Lakshmi Muthukrishnan* and Radhika Raman \\ *Correspondence: vcmlck@yahoo.co.in \\ Kanchi Kamakoti CHILDS Trust Hospital, CHILDS Trust Medical Research Foundation, Chennai, Tamil Nadu, India.
}

\begin{abstract}
Objective: To analyze the indications for chest (CXR) radiography, radiological abnormalities and the clinical predictors for pneumonia in children who present with acute wheeze to the pediatric emergency department.

Methods: This prospective study was conducted in the emergency department of a tertiary care pediatric referral hospital between July and December 2012. Children between 6 months to 18 years of age presenting with acute wheeze were included in the study.

Results: Of a total of 126 children included in the study, the most common indication for CXR was temperature $>100^{\circ} \mathrm{F}$ at presentation (65\%). The most common radiological abnormality was prominent bronchovascular markings in $67 \%$. Ten children $(8.7 \%)$ had radiological evidence of pneumonia, seven of them were $<5$ years of age. Presence of fever during triage $(\mathrm{p}=0.006)$, hypoxia $(p=0.01)$ and localised chest findings on auscultation $(\mathrm{p}=0.001)$ were statistically significant clinical predictors for pneumonia.

Conclusion: The incidence of radiographically confirmed pneumonia among children with wheezing is uncommon. Definite clinical criteria should be defined to avoid unwarranted chest radiography in children with acute wheeze.
\end{abstract}

Keywords: Acute, wheeze, children, pneumonia, chest radiography

\section{Introduction}

Wheeze is a common paediatric emergency. Children with viral respiratory infection often present with fever and acute wheeze and a chest radiograph is often performed as it is difficult to identify pneumonia by clinical examination alone.

Identifying clinical parameters which are more likely to be associated with pneumonia in children who present with acute wheeze can possibly prevent unwarranted radiography.

\section{Aim}

The aim of our study was to analyse the radiological abnormalities, indications for chest radiography and the clinical predictors for pneumonia in children who present with an acute wheeze to the paediatric emergency department.

\section{Materials and methods}

This prospective study at the emergency department of a tertiary care paediatric referral hospital was conducted between July to December 2012. Children who presented with acute wheeze aged between 6 months to 18 years were included. Children with chronic lung disease, history of tracheostomy, complex congenital heart disease, underlying immunodeficiency and malignancy were excluded from study. The decision to obtain a chest $x$ ray $(C X R)$ was left to the discretion of pediatric post graduates, fellows in emergency medicine, registrars and paediatric consultants in the emergency room who was blinded to the ongoing study. Clinical parameters (Table 1) such as fever, chest retractions, tachypnea, focal lung signs, oxygen saturation $\left(\mathrm{SpO}_{2}\right)$, age, the need for intensive care unit admission and findings on chest radiography were continually abstracted from medical records throughout the study period. Severity of wheeze was determined by the Pulmonary score index (PSI) [1]. PSI $0-3$ was mild, $4-6$ moderate and $>6$ was considered as severe wheeze. The variables included in pulmonary score index are respiratory rate, wheeze and accessory muscle activity. All radiographs were reviewed by a qualified pediatric radiologist for the presence (as evidenced by consolidation) or absence of pneumonia.

\section{Results}

A total of 126 children were included the study of which $70 \%$ were males and $73 \%$ were between $1-5$ years of age. Ninety two children (73\%) had a PSI score of $4-6$. Children with PSI > $6(n=13)$ required admission to the intensive care unit. Of the 115 children who had a CXR, sixty had previously undergone chest radiography for wheeze $(n=52$, single $\& n=8$, multiple times). On analysis it was found that $65 \%$ of children had a CXR as they were febrile. The other indications being $1^{\text {st }}$ episode of wheeze (25\%) suspected foreign body $(5 \%)$, persistent hypoxemia (3.5\%) and suspected cardiac pathology $(0.85 \%)$ (Figure 1). Radiological abnormalities (reported were as follows, prominent bronchovascular markings (67\%), hyperaerated lungs (21\%), consolidation ( $8.7 \%)$, atelectasis (3.5\%) and foreign body (0.8\%) (Figure 2). Out of 10 children (8.7\%) who had radiological evidence for pneumonia majority $(n=7)$ were in the age group of 1 to 5 years. The clinical predictors for pneumonia were triage temperature $=$ or $>101^{\circ} \mathrm{F}(10 / 10)$, hypoxia (4/10), localised chest findings (7/10) (Figure 3A-3C). Chi 
MuthuKrishnan et al. Pulmonology \& Respiratory Research 2013,

Table 1. Demographic characteristics of the study population $(\mathrm{N}=126)$.

\begin{tabular}{lc}
\hline Patient Characteristics & n (\%) \\
\hline Age & $26(21)$ \\
$<1 \mathrm{yr}$ & $92(73)$ \\
$1-5 \mathrm{yr}$ & $8(6.3)$ \\
$>5 \mathrm{yr}$ & $87(70)$ \\
Male & $60(48)$ \\
History of wheezing & \\
Triage temperature & $14(11)$ \\
$\geq 101^{\circ} \mathrm{F}$ & $61(48)$ \\
$<101^{\circ} \mathrm{F}$ & \\
\hline Pulmonary Score Index (PSI) & $13(10)$ \\
$0-3$ (mild) & $89(71)$ \\
$4-6$ (moderate) & $24(19)$ \\
$>6$ (severe) & 13 \\
Admission in PICU & 11 \\
Admission in ward & \\
\hline Triage oxygen saturation & $17(13)$ \\
$\leq 92 \%$ & $109(87)$ \\
$>92 \%$ & $13(10)$ \\
Localised chest findings & $115(91)$ \\
Children who had CXR & \\
\hline Children who had previous CXR & \\
Once & \\
Multiple & \\
Radiographic pneumonia & \\
\hline
\end{tabular}

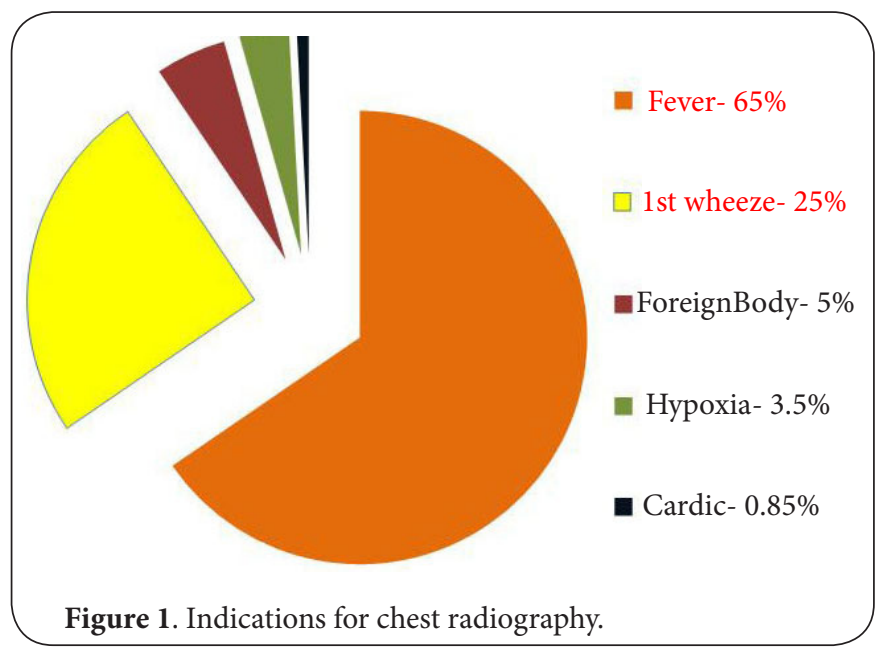

square test was applied and $p$ value of $<0.05$ was considered significant. Presence of fever $(p=0.006)$, hypoxia $(p=0.01)$ and localised chest findings $(p=0.001)$ were statistically significant (Table 2). PSI of more than 6 was not associated with pneu-monia.

\section{Discussion}

The criteria for ordering a chest $x$ ray for children with acute wheeze are not well defined. Chest $x$ rays are performed frequently in children who present with acute wheeze, as it is difficult to rule out pneumonia based on history and clinical examination alone. Pneumonia in children with acute wheeze is uncommon and imaging adds to the health
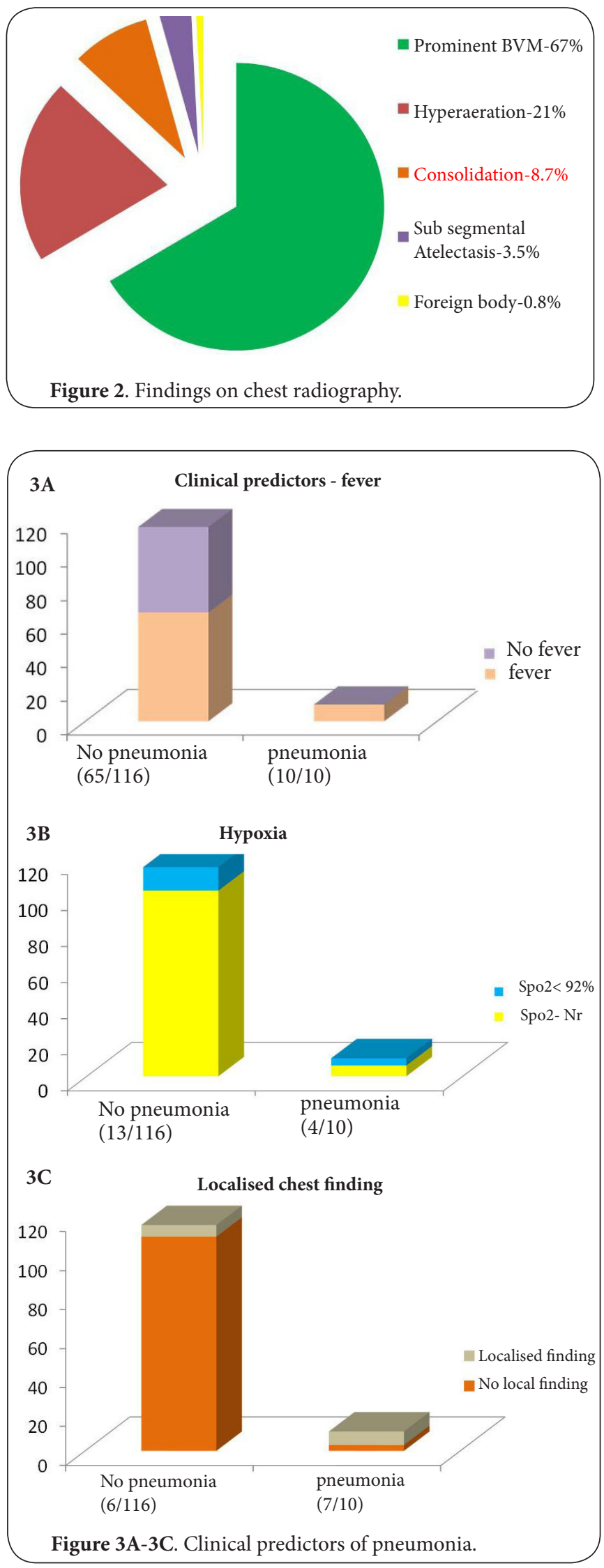
MuthuKrishnan et al. Pulmonology \& Respiratory Research 2013, http://www.hoajonline.com/journals/pdf/2053-6739-1-1.pdf

Table 2. Parameters predictive of pneumonia.

\begin{tabular}{lccc}
\hline Variables & $\begin{array}{c}\text { No Pneumonia } \\
(\mathbf{n}=\mathbf{1 1 6})\end{array}$ & $\begin{array}{c}\text { Pneumonia } \\
(\mathbf{n}=\mathbf{1 0})\end{array}$ & $\begin{array}{c}\text { P value By Chi Square Test } \\
\mathbf{P}<\mathbf{0 . 0 5}=\text { Significant }\end{array}$ \\
\hline Temp $\geq 101^{\circ} \mathrm{F}$ & 65 & 10 & 0.006 \\
Hypoxia & 13 & 4 & 0.01 \\
Localised chest findings & 6 & 7 & 0.001 \\
Moderate PSI score & 82 & 7 & 0.75 \\
\hline
\end{tabular}

care cost and exposure of children to ionizing radiation all of which can be avoided. Analysis of CXR taken for children with acute exacerbation of wheeze in the emergency department suggests that in most instances it does not alter the diagnosis or management. We found a low rate $(8.7 \%)$ of radiographic pneumonia in our study population which is comparable to $4.9 \%$ in a study from Boston [2]. The prevalence of pneumonia in previous investigations varied widely, ranging from $8.6 \%$ to $35 \%$ [3-5].

In our study all children (100\%) who had radiological evidence for pneumonia had triage temperature $\geq 101^{\circ} \mathrm{F}(\mathrm{p}=0.006)$. A study from Pakistan also concludes that temperature of $>100^{\circ} \mathrm{F}$ was highly specific for poor response to bronchodilator and susequent deterioration. The overall positive predictive value was best for temperature of $>100^{\circ} \mathrm{F}$ [6]. We also observed in our study that in addition to temperature, hypoxia $(p=0.01)$ and localised chest findings $(p=0.001)$ were also statistically significant variables associated with pneumonia. The difference in Pulmonary score index in children without radiological pneumonia was not stastically significant as PSI mainly includes respiratory rate, wheeze and work of breathing. This observation is similar to a prospective study which reported that, among infants $<18$ months of age, grunting on examination and oxygen saturation of $<93 \%$ were predictors of radiographic pneumonia, whereas fever and tachypnea were not associated with pneumonia risk [7]. In a study from Brazil, absence of pulmonary infiltrates was associated with the complaint of difficulty in breathing $(P=0.04)$ and wheezing $(P=0.001)$ [8]. It is also difficult to distinguish a viral process from bacterial pneumonia on chest radiographs, and radiographic pneumonia does not necessarily indicate a bacterial infection leading to overuse of antibiotics.

\section{Conclusion}

Majority of children with acute wheeze are subjected to chest radiography as they are febrile, though the occurrence of radiographic pneumonia is as low as (8.7\%). Triage temperature of $\geq 101^{\circ} \mathrm{F}$, hypoxia and localized chest findings were predictors of pneumonia in children with acute wheeze. Definite criteria have to be defined through larger studies to avoid unnecessary chest radiography.

\section{Competing interests}

The authors declare that they have no competing interests.

\section{Authors' contributions}

Lakshmi Muthukrishnan did the collection, analysis of data and preparation of manuscript. Radhika Raman conceived, designed and critically analysed the study.

\section{Acknowledgement}

S. Muralinath, Consultant radiologist.

Publication history

Editor: Chunbin Zou, University of Pittsburgh, USA.

EIC: Victor J. Thannickal, University of Alabama at Birmingham, USA. James R. Seibold, Scleroderma Research Consultants, LLC, USA.

Received: 13-May-2013 Revised: 13-Jun-2013

Accepted: 21-Jun-2013 Published: 11-Jul-2013

\section{References}

1. Smith SR and Strunk RC. Acute asthma in the pediatric emergency department. Pediatr Clin North Am. 1999; 46:1145-65. | Article | PubMed

2. Mathews B, Shah S, Cleveland RH, Lee EY, Bachur RG and Neuman MI. Clinical predictors of pneumonia among children with wheezing. Pediatrics. 2009; 124:e29-36. | Article | PubMed

3. Leventhal JM. Clinical predictors of pneumonia as a guide to ordering chest roentgenograms. Clin Pediatr (Phila). 1982; 21:730-4. | Article | PubMed

4. Lynch T, Gouin S, Larson C and Patenaude Y. Does the lateral chest radiograph help pediatric emergency physicians diagnose pneumonia? A randomized clinical trial. Acad Emerg Med. 2004; 11:625-9. | Article | PubMed

5. Mahabee-Gittens EM, Grupp-Phelan J, Brody AS, Donnelly LF, Bracey $\mathrm{SE}$, Duma EM, Mallory ML and Slap GB. Identifying children with pneumonia in the emergency department. Clin Pediatr (Phila). 2005; 44:427-35. | Article I PubMed

6. Hazir T, Qazi S, Nisar YB, Ansari S, Maqbool S, Randhawa S, Kundi Z, Asghar R and Aslam S. Assessment and management of children aged 1-59 months presenting with wheeze, fast breathing, and/or lower chest indrawing; results of a multicentre descriptive study in Pakistan. Arch Dis Child. 2004; 89:1049-54. | Article | PubMed Abstract I PubMed Full Text

7. Mahabee-Gittens EM, Dowd MD, Beck JA and Smith SZ. Clinical factors associated with focal infiltrates in wheezing infants and toddlers. Clin Pediatr (Phila). 2000; 39:387-93. | Article | PubMed

8. Key NK, Araujo-Neto CA, Cardoso M and Nascimento-Carvalho CM. Characteristics of radiographically diagnosed pneumonia in under-5 children in Salvador, Brazil. Indian Pediatr. 2011; 48:873-7. | Pdf | PubMed

\section{Citation:}

Muthukrishnan L and Raman R. Analysis of clinical \& radiological findings in children with acute wheeze.

Pulmonol Respir Res. 2013; 1:1.

http://dx.doi.org/10.7243/2053-6739-1-1 